\section{Teaching and Learning Science through Multiple Representations: Intuitions and Executive Functions}

\author{
Janice Hansen ${ }^{\dagger}$ and Lindsey Engle Richland ${ }^{\ddagger *}$ \\ ${ }^{\dagger}$ Director of Undergraduate Education and ${ }^{\ddagger}$ Associate Professor of Education, School of Education, \\ University of California Irvine, Irvine, CA 92697
}

\begin{abstract}
Reasoning about visual representations in science requires the ability to control one's attention, inhibit attention to irrelevant or incorrect information, and hold information in mind while manipulating it actively-all aspects of the limited-capacity cognitive system described as humans' executive functions. This article describes pedagogical intuitions on best practices for how to sequence visual representations among pre-service teachers, adult undergraduates, and middle school children, with learning also tested in the middle school sample. Interestingly, at all ages, most people reported beliefs about teaching others that were different from beliefs about how they would learn. Teaching beliefs were most often that others would learn better from presenting representations one at a time, serially; while learning beliefs were that they themselves would learn best from simultaneous presentations. Students did learn best from simultaneously presented representations of mitosis and meiosis, but only when paired with self-explanation prompts to discuss the relationships between the graphics. These results provide new recommendations for helping students draw connections across visual representations, particularly mitosis and meiosis, and suggest that science educators would benefit from shifting their teaching beliefs to align with beliefs about their own learning from multiple visual representations.
\end{abstract}

\section{INTRODUCTION}

Science, even in a defined field of study such as biology, is not a set of discrete facts, but an interconnected system of complex concepts. The development of conceptually organized and integrated scientific knowledge is an overarching goal of science education, articulated at the K-12 level in the United States recently within the Next Generation Science Standards (NGSS Lead States, 2013). One of the most common instructional supports that science teachers use to engage students in thinking about complex relationships is sequences of visual representations such as diagrams, pictures, or animations (Roth et al., 2006). Visualizations are central to the learning of science, the practice of science, and the communication of science, and both cognitive scientists and educators agree that they are a vitally important component of science teaching (Ainsworth and Newton, 2014; Eilam and Gilbert, 2014; Matthewson, 1999). Multiple representations can help learners construct deeper understanding of scientific concepts or system structures than single representations used alone (National Research Council, 2012; Ainsworth, 2014). These may be particularly useful when concepts are complex and interrelated, as is the case when one concept builds on another. As learners compare and contrast graphics, they are better able to construct deeper domain understanding (Ainsworth, 2014).

At the same time, one representation is rarely adequate to capture the entirety of a science concept, and multiple representations are often used to describe related aspects of a system (e.g., a diagram of a heart and a diagram of a circulatory system; see
Ido Davidesco, Monitoring Editor

Submitted Nov 19, 2019; Revised Sep 14, 2020; Accepted Sep 29, 2020

CBE Life Sci Educ December 1, 2020 19:ar61 DOI:10.1187/cbe.19-11-0253

*Address correspondence to: Lindsey Richland (l.richland@uci.edu; uciscienceoflearning.org). (C) 2020 J. Hansen and L. Richland. CBE-Life Sciences Education @ 2020 The American Society for Cell Biology. This article is distributed by The American Society for Cell Biology under license from the author(s). It is available to the public under an Attribution-NoncommercialShare Alike 3.0 Unported Creative Commons License (http://creativecommons.org/licenses/ by-nc-sa/3.0).

"ASCB®" and "The American Society for Cell Biology ${ }^{\circledR}$ " are registered trademarks of The American Society for Cell Biology. 
Roth et al., 2006). Concerns have been raised, however, about the potential of multiple representations to overly tax learners' cognitive resources, leading them to not be able to fully process or reason on the basis of the information provided or notice and make inferences about the relationships between the representations (Kirschner, 2002; Cho et al., 2007). This is particularly problematic when the relationship between the representations is important, such as in the case of the model of a heart and a model of the human circulatory system with the heart at its center. Similarly, when two diagrams are intended to allow learners to compare and/or contrast aspects of systems, for example, comparing diagrams of cellular reproduction between those undergoing mitosis and meiosis, both visual representations capture important information that should be learned, but the differences between them are also illustrative and conceptually important.

Adding to the complexity of using multiple representations in classrooms are teacher and student beliefs about how people learn best from these tools. Teachers hold private beliefs about subject matter, teaching, and learning, and these influence their teaching practices (National Academy of Sciences, 2000). Learners also have beliefs about how they learn best, though they are not always reliable judges of their own learning from different classroom practices. This is particularly true when individuals are required to put forth extra effort in learning (Deslauriers et al., 2019). Understanding these naïve belief systems and how they align with instructional outcomes may inform the development of more effective practices for using multiple representations in classrooms.

This article draws on the cognitive science literature to provide a novel lens for understanding the challenges inherent in learning biological science from the relationships between representations. Cognitive scientists have widely demonstrated that the human attentional system has limited resources, such that one can only meaningfully and actively process a limited set of information at once (see Baddeley and Hitch, 1974; Engle, 2002; Miyake and Friedman, 2012; Diamond, 2013). Generally, this system has been described as a system of executive functions (EFs), which are comprised of three broad components that enable a person to selectively allocate attention to information in the world and that are correlated but distinct processes (Miyake et al., 2000; Miyake and Friedman, 2012). Working memory (WM) is defined most broadly as the ability to hold information in mind and manipulate it (see Baddeley and Hitch, 1974; Engle, 2002; Miyake and Friedman, 2012). Working memory is not simply the ability to hold information in mind (e.g., a list of vocabulary words) but also to do cognitive work with that information (e.g., reorganizing new vocabulary words into a concept map). Inhibitory control (IC; see Diamond, 2013) is described as the integrated processes of inhibiting attention and prepotent actions based on irrelevant or misleading information (e.g., saying "night" when presented with a diagram of a sun, or expending effort to not consider the size of a textbook drawing of a cell to avoid misconceptions that cells are visible to the natural eye). Task switching refers to the processing involved in changing one's goal-oriented task engagement and routines, for example, switching categorization criterion or switching from pointing to parts of a cell diagram to explaining how cells are part of a reproductive system (see Miyaki et al., 2000). While separate processes, EFs are generally believed to share a limited set of resources, such that if someone is exerting all of their cognitive resources attempting to inhibit attention to something very salient but misleading, they will have less capacity to use WM to make inferences about the relationships between representations.

EFs are primarily controlled by the prefrontal cortex of the brain, an area that has been found to develop well into adolescence (Diamond, 2013). Therefore, when reasoning about complex relationships between visual representations, which has a high requirement for EFs (see Waltz et al., 2000; Simms et al., 2018), children may need significant support for noticing key correspondences of visual representations and ignoring irrelevant or misleading features. This is particularly the case when reasoning about complex concepts such as scientific systems or solving complex problems (e.g., Gick and Holyoak, 1980, 1983; Zook, 1991).

Though using diagrams, charts, pictures, and models is common classroom practice, careful consideration of the psychological processes of learning will aid educators in optimizing student learning from these visual representations. Theories of learning from multiple representations have generally focused on how to engage cognitive resources effectively and avoid high demand that is not intrinsic to the conceptual aspects of the intended task (Sweller et al., 1998; Mayer, 2019). Theories of multimedia learning build on this foundation to describe how learners make sense of text and pictures presented together. While well-designed instruction that includes text and media together may enhance learning, the processes whereby learners make sense of both textual and visual input within the cognitive architecture are complex (for a full discussion, see Mayer, 2019) and involve the coordination of more than one cognitive subsystem (Schnotz and Bannert, 2003; Schnotz, 2019). Complementary representations, which are designed to highlight comparisons across diagrams, as in the case of the related processes of photosynthesis and respiration, may be particularly difficult for learners to process (Ainsworth, 2014).

\section{The Role of EFs in Making Sense of Visual Representations} Cognitive scientists broadly agree that the complexity and amount of information to be processed in visual representations can be cognitively demanding, particularly when the relationships between representations are meaningful and will lead the learner to build a broader understanding of the concept being represented (Phillips et al., 2010). The optimal way to reduce the burden on learners has been explored, yet not fully answered, and the relationship between research and practice remains complex (Ainsworth and Newton, 2014).

One field of cognitive science focuses on relational and analogical reasoning, exploring how reasoners draw connections between representational systems (see Holyoak, 2012). Some have argued that the process of drawing structural (or conceptual) relationships between representations imposes a high burden on WM and IC of attention, particularly when the representations are not visible simultaneously (see Cho et al., 2007; Krawczyk et al., 2008; Begolli et al., 2018).

One can compare the surface and structural elements of visual representations. Surface-level elements are those that are based in the appearance of the figures (e.g., the colors, shapes, and sizes of the objects). The structural or relational elements 
are the relationships between and among the visual forms, which are more typically the abstract scientific processes being explained (e.g., cell reproduction in a diagram of mitosis). As understood within the structure-mapping theory of analogical reasoning, reasoners make inferences by taking a mental model of the key structured relationships within one representation and aligning them with the key structures within a target problem, concept, or representation. They may notice the surface appearance of the visual representations, and sometimes those provide clues about how to align and recognize abstract relationships across the representations, but those surface features are typically not intended to be what was memorized. Instead, learners should map correspondences between those aligned representations to notice key abstract/conceptual similarities or differences and then draw inferences based on those alignments about the target context.

When the related representations are presented one at a time-serially - this alignment process is more effortful. When looking at the second figure, the reasoner must recall a prior visual representation and hold it in WM, while manipulating it to determine its relevance to the currently visible visual representation. The complexity involved in this mental processing is clear when considering related science concepts such as mitosis and meiosis, where a learner might align the structures of chromosomal replication, the process of cellular division, the characteristics of daughter cells, and so on, to construct an understanding of how the two types of cellular reproduction relate to each other. The learner may first notice that representations of mitosis and meiosis often both contain circles that show cells, and each cell has some wiggly lines (chromosomes) inside (i.e., the surface features of these representations), but this is not the key insight; rather, learners must go further to notice the relationships. They must see the changes from one cell to the next and the relative numbers of chromosomes in particular.

Importantly, the relational reasoning literature suggests that having visual information available should reduce the burden on reasoners' cognitive processing by providing WM off-load, yet at the same time, this may not be enough to ensure that reasoners notice and map correspondences across representations (Gick and Holyoak, 1983). Thus, reasoners may require additional support to draw their attention to link the representations actively. This may be especially important when the burden on EF for representing the information is high (Richland and McDonough, 2010; Begolli and Richland, 2016). In a classroom context, children's individual level of EF capacity predicted learning from a lesson in which visual representations were not visible simultaneously (Begolli et al., 2018), providing some evidence that, particularly for children with lower levels of EF available, ensuring that multiple representations are visible simultaneously and well supported might be important for improving learning.

In contrast, cognitive scientists within the field of multimodal learning, drawing on Baddeley and Hitch's (1974) model of WM, as well as cognitive load theory (see Sweller et al., 1998), have argued that simplifying representations is important to reducing cognitive overload and improving intended learning (for a comprehensive review of historical visual representation research, see Phillips et al., 2010). In particular, Mayer's cognitive theory of multimedia learning (see Mayer, 2019) suggests that, when a person processes a visual representation with text, he or she develops two mental representations of the material. One mental representation draws on resources within the verbal WM system based on the text, and the other draws on resources within a visual-spatial WM store, which Baddeley's model of WM suggests draws on the same overall WM resources. So, if a visual representation has too much information of both types, a learner's attentional resources might be overloaded, leaving little EF stores to inhibit irrelevant information (e.g., irrelevant colors and graphics to promote interest). More importantly to our current discussion, this might also impede processing of the relationships between representations. The theory does hypothesize that providing language and visual representations simultaneously can improve overall knowledge, but suggests that auditory narration is better than written text when possible. Thus, it is not clear how one would optimize learning from multiple representations when the instructional goal is to have students compare these, recognize correspondences, recognize differences, or otherwise relate them. The field of multimodal learning and cognitive load suggests that presenting two visual representations with accompanying text labels, all simultaneously, might be too high a burden for the EF system.

Thus, while science educators generally agree that guiding students toward an organized, complex, richly connected understanding of science topics through multiple visual representations is desirable (Ainsworth and Newton, 2014), implementing this type of instruction requires careful consideration of the cognitive processes involved, and the implications for sequencing presentation of such representations are not yet clear. Classroom instruction that effectively supports children in making deep conceptual connections is challenging for teachers (e.g., Stein et al., 2008; Smith et al., 2009), so more information about these details of instruction have important applied implications.

\section{The Current Studies}

The studies reported in this article explore teachers' and learners' intuitive beliefs about learning from visual representations of related science concepts (Study 1) and tests those beliefs experimentally in a computer-based classroom lesson with middle school students (Study 2). Learning from multiple representations is a key pedagogical consideration in teaching science for reasoning (NGSS Lead States, 2013) and raises questions about whether optimal learning emerges from two representations displayed simultaneously, where EF resources could be focused on drawing connections and generating inferences, or displayed serially, where EF resource demands on processing each representation would be reduced.

This study focused on the two processes of cellular reproduction: mitosis and meiosis. This is a critical component of most introductory biology curricula and one that every high school student must master (NGSS Lead States, 2013). While mastery of these topics requires more depth than introduced here, this is an ideal pedagogical context for examining intuitions and learning from multiple representations, as these two processes are highly related but have core structural differences that are regularly made visible through diagrammatic representations. Thus, the findings here will have direct implications for teaching this core biological topic and also will provide insights for any of the many pedagogical contexts in the sciences where representations are necessary to support students in building 
from the understanding of one case to a second with related but different structure. This might include comparing representations of plant and animal cells or, at a more complex level, anatomical systems such as respiratory and circulatory systems.

The first study explored implicit beliefs of pre-service teachers, adult non-educators who were currently undergraduates, and middle school children, regarding whether people learn better from multiple visual representations that are presented simultaneously or serially and the reasons they gave for holding these beliefs. The three groups were asked how they themselves learned best, as well as how they believed a younger set of learners would learn best to distinguish between their understanding of their own cognition and their beliefs based on their own naïve theories of pedagogy. The primary research questions were whether these two sets of learning beliefs would diverge, assessing whether there were differences between peoples' beliefs about their own learning and their beliefs about pedagogy-how others would learn best.

The second study tested how these implicit beliefs related to the mental representations children gained from instruction that involved comparisons between multiple representations with lower or higher levels of support for drawing connections between them, including serial versus simultaneous presentation, as well as more explicit prompts to actively align and compare the representations. To answer these questions, we developed an experiment wherein children were randomly assigned to learn from a computerized instructional module in which only the method of presenting diagrams varied across conditions.

The preponderance of research regarding how people process multiple representations has been conducted with adults rather than children (Cook, 2006). This is an important oversight, given that visual representations are the most commonly used instructional supports in American K-12 science classes (Roth et al., 2006). This study aimed to test predictions made by the cognitive science literature regarding best practices for supporting students in learning from multiple representations. We tested both the mode of ordering the representations (sequentially vs. simultaneously), as well as the level of pedagogical support provided. Thus, Study 1 and 2 together allowed us to gain insight into adults' and youths' teaching beliefs about ordering multiple representations and the alignment between these beliefs and students' actual learning.

\section{STUDY 1: SURVEY OF IMPLICIT BELIEFS ABOUT LEARNING FROM MULTIPLE REPRESENTATIONS}

While both cognitive scientists and science teachers agree that visual representations are important tools in teaching science, the alignment of research to teaching practice is not always direct, and in the demands of real classroom practice, teachers rely heavily on personal judgment in deciding what visual representations to use and how they will be presented (Ainsworth and Newton, 2014). Yet little is known about what informs these judgments, particularly when it comes to multiple representations of related science concepts.

One concern that teachers have is about student competency in interpreting visual representations (Eilam and Gilbert, 2014). But students are not blank slates when they approach a diagram or picture. They have their own metacognitive beliefs about how they learn best. Students, however, are not always the best judges of what helps them learn, particularly when it comes to passive versus effortful learning (Deslauriers et al., 2019).

The metacognitive beliefs people hold can influence what and how they can learn (Greeno et al., 1996; Pamuk et al., 2016). Teacher beliefs about learning can influence their classroom practices of teaching, and in turn, indirectly affect student achievement (Muijs and Reynolds, 2015). Further, beginning science teachers and experienced science teachers can have different views of their role in helping students learn. Beginning teachers may hold more teacher-centered traditional views of delivering information (Luft and Roehrig, 2007). Part of building learning theory in educational contexts involves understanding the teaching and learning beliefs that teachers bring to the classroom. Here we collect data to understand beliefs about teaching and learning expressed by pre-service teachers, adult non-educators, and children. We specifically focus on the pedagogical context of how to best sequence visual presentations of multiple representations-simultaneous versus serial presentation.

\section{Methods}

Participants. The survey sample included 89 pre-service teachers, 211 adult non-educators, and 385 middle school children. The 89 pre-service teachers were enrolled in a combined credential/master of arts in teaching program at a large suburban university. They were enrolled in a basic cognition class but had not yet received any explicit instruction about either EFs or the use of visual representations. The 211 adult non-educators were undergraduate students at the same university and represented a wide variety of different majors. All adult participants consented to participation in the study. The 385 middle school students were seventh-grade students of three science teachers at two different suburban schools from the same upper-middleclass district. The middle school participants were recruited through their science teachers. The day before the study, students were given a letter to take home that described the study. The letter informed parents/guardians that students were not required to participate, and that no student-identifiable data would be collected. On the day of data collection, students were read a description of the study and indicated assent through the raising of hands. Students were informed that they could remove themselves from the study at any time and receive the same instruction through text-based instruction provided by their teacher. One student opted out of the study and was not included in further analyses.

Materials and Procedure. The surveys administered to each population were slightly different in framing due to the different educational background of the children, adults, and teacher candidates, but the key questions analyzed in this study were the same. Also, we intentionally asked each population about their beliefs of how a younger population would learn best, so this differed across participant groups.

Adult participants were given a pencil-and-paper survey as part of a larger, unrelated study. Participants were told that their responses would be used to inform development of new science teaching materials. They were asked about their own science backgrounds and whether they felt they could describe the related processes of mitosis and meiosis to a friend. They were then given a forced-choice item that asked whether they 
TABLE 1. Reasons given for preferring serial or simultaneous presentation

\begin{tabular}{|c|c|c|c|}
\hline Code & $\begin{array}{c}\text { Adult sample } \\
\text { kappa }\end{array}$ & $\begin{array}{c}\text { Child sample } \\
\text { kappa }\end{array}$ & Participant sample responses \\
\hline Ability to compare and contrast & 0.90 & 0.95 & $\begin{array}{l}\text { That way I could compare them; This way I can see what is the same and what } \\
\text { is unique. }\end{array}$ \\
\hline Described as "easier" & 0.89 & 0.86 & $\begin{array}{l}\text { It's easier to learn like that; It's too hard if you have to remember one thing } \\
\text { and go back and remember something else; it's easier for kids to focus on } \\
\text { one thing at a time. }\end{array}$ \\
\hline
\end{tabular}

thought they would learn better from a combined (simultaneous presentation) diagram of mitosis and meiosis, or if they thought it would be better to learn from separate diagrams (presented serially). A free-response question asked them to justify their choices. A follow-up question asked them to predict how a middle school student would learn better, and again, participants were asked why they made that choice. The entire survey took approximately 5 minutes to complete, and the key questions are available in the Supplemental Material.

Middle school students answered the same basic questions as the adults, but as they had not yet been exposed to instruction about mitosis and meiosis, the forced-choice item asked whether they would prefer to learn about the related topics of animal and plant cells through representations of each cell presented simultaneously or serially, and why. This was followed up by items asking how they thought a fourth-grade student would learn best and why.

Measures and Data Coding. Forced-choice responses asking how people would best learn from related diagrams were simply coded "simultaneous" or "serial." For free-response items asking participants why they made the choice they did, categorical codes were developed to quantify data for comparison. These codes were developed through an iterative process informed by EF literature on learning and its relationship to comparing and contrasting representations (e.g., Krawczyk et al., 2008; Holyoak, 2012; Begolli et al., 2018) and refined by the responses themselves. Interrater reliability across codes was set at kappa $\geq 0.80$. Two raters coded a training data set of student responses to allow for discussion and resolution of any discrepancies in codes assigned. The two raters then independently coded $20 \%$ of each data set to attain reliability. A single rater (J.H.) coded the remaining data independently. The coding manual is available in the Supplemental Material.

Four codes scored responses to items that asked why people would learn best from either simultaneous or serial presentation of visual representations. These codes were 1) ability to compare and contrast; 2) promotes deeper understanding; 3) described as easier or not as difficult; and 4) cites reducing confusion as a goal. The codes were not mutually exclusive, and a response could receive more than one code. The differences between each of these codes rested on the participants' explicit use of words that highlighted each of these ideas (e.g., to compare or to reduce confusion) or a clear framing that allowed a coder to differentiate their intention. "Easier versus difficult" provided only a graded description of difficulty and was coded separately from any statements regarding confusion as a mechanism that would form the source of any difficulty. These codes (reported with their associated kappa statistics) are detailed in Table 1.

\section{Results}

Beliefs results are provided for the three sets of participants separately, with overall means and results shown in Figure 1. Each is discussed in turn, analyzing frequency of endorsing simultaneous versus serial representations both for their own learning and for teaching others who were younger than themselves.

Pre-service Teachers. Pre-service teachers $(n=89)$ did not overwhelmingly endorse one way of presenting conceptually related visual representations for their own learning, with beliefs split between simultaneous and serial presentation orders as optimal. This difference was not significant; $\chi^{2}(1, N=$ $89)=0.91, p=0.34$. Interestingly, their reasons for selecting each of these two different orders were different. Among teachers who endorsed serial presentation of related diagrams for their own learning, avoiding confusion was the most often cited reason ( $n=17,34.7 \%)$. Those who said they preferred related diagrams presented simultaneously cited the ability to compare and contrast as the reason this was desirable ( $n=34,85.0 \%)$.

When asked how middle school students would learn best, however, pre-service teachers significantly often changed their beliefs, and indicated that the learning needs of middle school students differed from their own; $\chi^{2}(1, N=89)=22.48, p<0.01$. As shown in Figure 1, most indicated that serial presentation would be optimal for middle school students. This suggests that the pre-service teachers held a tacit belief that there is a developmental difference in the learning needs of middle school students versus adults when analyzing multiple representations.

The reasons preservice teachers gave for these decisions are similar to those described for how they would themselves learn. Of the 59 respondents who said middle school students would learn best from serially presented diagrams, the most-cited reasons for endorsing this style were avoiding confusion $(n=22$, $37.2 \%)$, ease of interpretation $(n=15,25.4 \%)$, and promoting deeper understanding $(n=11,18.6 \%)$. Of the 30 pre-service educators who said middle school students would learn best from simultaneously presented diagrams, $80.0 \%(n=24)$ cited the ability to compare and contrast as the reason why this method was preferable.

Adult Non-educators. In contrast to pre-service teachers, the adult non-educators, who were currently undergraduate students, indicated a clear preference for simultaneous presentation for their own learning; $\chi^{2}(1, N=211)=50.28, p<0.01$. 


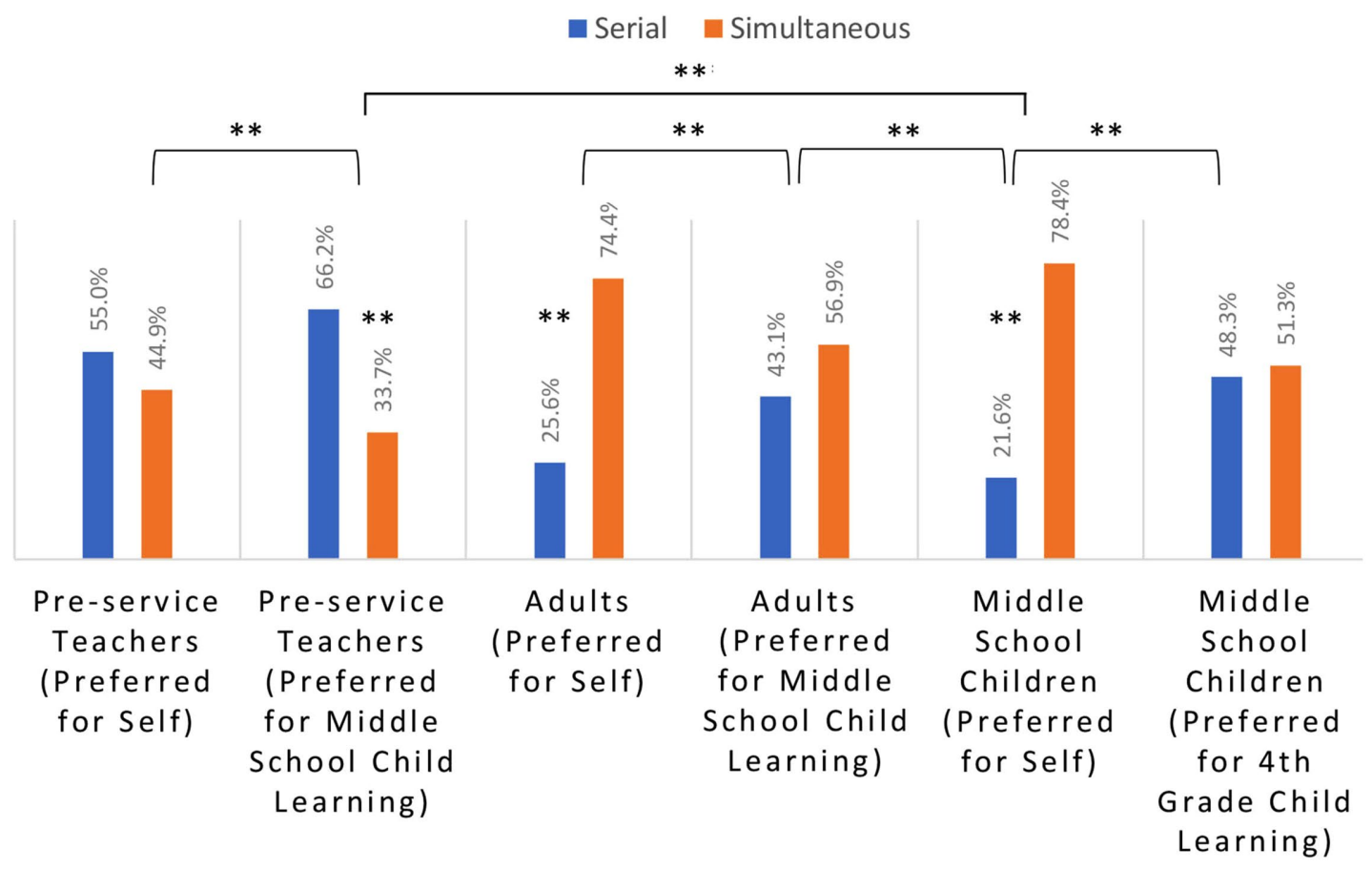

$* *$ denotes significant difference, $p<.01$

FIGURE 1. Adult, pre-service teacher, and middle school student beliefs about optimal presentation of multiple representations for one's own learning versus instruction of others.

Again, the reasons for endorsing simultaneous ordering were the same. Among the 54 participants who endorsed serial presentation for their own learning, the most common reasons cited were ease of interpretation ( $n=23,42.6 \%)$, and avoiding confusion ( $n=22,40.7 \%)$. Of the 157 people who preferred simultaneous presentation, $89.2 \%$ cited the ability to compare and contrast as the reason $(n=140)$.

When asked whether serial or simultaneous visual representations were preferable for middle school students, like pre-service teachers, a significant number of adult non-educators felt middle school students would benefit from a different manner of presentation than themselves as adults; $\chi^{2}(1, N=211)=$ 21.96, $p<0.01$. This shifted to more recommendations for serial presentation for children than for themselves, though there were not significant differences between these two; $\chi^{2}(1$, $N=211)=3.99, p=0.05$.

The main reason one style of presentation was preferred over the other was similar for the adult non-educators as for the pre-service teachers. For those endorsing serial presentation, avoiding confusion was cited by 48 of the 91 respondents (52.7\%). Among 120 people who felt simultaneous presentation would be better, 89 (74.2\%) cited the ability to compare and contrast as important.

Middle School Students. Like their adult counterparts, middle school students had strong opinions about the way related visual representations should be presented. Students of color in the proportion of participation as a result of the transition online. As shown in Figure 1, simultaneous presentation was preferred for their own learning. This difference was significant; $\chi^{2}(1, N=385)=124.57, p<0.01$. Again of interest is that these youth cited the same reasons for preferring simultaneous versus serial presentation order as the adults did. Those who preferred serial presentation endorsed its role in avoiding confusion (46.9\%, $n=39$ ), and the belief that it would lead to greater understanding $(24.1 \%, n=20)$. The significant reason for endorsing simultaneous presentation was the ability to compare and contrast $(86.4 \%, n=261)$.

As in adults, the children's beliefs about how they would learn best differed significantly from how they thought younger students would learn; $\chi^{2}(1, N=384)=19.02, p<0.01$. When asked what presentation would be better for fourth-grade students, the middle school students were split, with 197 (51.3\%) endorsing simultaneous presentation. Respondents who selected serial presentation were more likely than those selecting simultaneous presentation to say it would help younger children avoid confusion or distraction $(12.8 \%, n=24)$ and lead to greater depth of understanding $(7.0 \%, n=13)$. Those who selected simultaneous presentation were most likely to suggest that the ability to compare and contrast would be enhanced $(23.3 \%, n=46)$.

Importantly, the beliefs of middle school students about how they would learn best differed significantly from the beliefs pre-service teachers held about the students' learning needs. Middle school students strongly preferred simultaneous presentation for their own learning, but the pre-service teachers felt that the students would learn better from serial presentation; $\chi^{2}(1, N=474)=68.94, p<0.01$.

\section{Discussion}

Previous research has indicated that beliefs about learning are important for their influence on teacher instructional practice 
(Friedrichsen et al., 2011). Collected survey data from the 211 adult non-educator and 385 middle school student samples were fairly consistent, in that both groups preferred to learn from simultaneous presentation of visual representations when learning about conceptually connected science concepts. The 89 pre-service teachers differed in that they did not significantly choose one manner of presentation over the other for their own learning. All of the adults were more likely to prefer serial presentation for middle school students, though the students themselves strongly preferred simultaneous presentation. This difference was significant when comparing pre-service teacher preference for middle school student learning and the middle school student preferences.

For participants who were drawn to serially presented visual representations, concern about the amount of information to be processed was commonly expressed. A typical response reads, "With just one [simultaneously presented diagram] it might get jumbled together and confusing." Those who preferred simultaneous presentation were more likely to cite the ability to compare and contrast as being desirable. This suggests that, across all three groups, participants had a sense that the EF resources required to process simultaneously presented diagrams would be much higher, at least initially, than the cognitive demand of processing serially presented diagrams.

Pre-service teachers felt strongly that middle school students needed serial presentation of diagrams of conceptually related content in order to learn best, while the responses of non-educators were evenly split between endorsing serial and simultaneous presentation. As one teacher pointed out, "Two diagrams would keep each process separate. This would help students get a clear idea of both processes before they are shown together." Non-educator adults and middle school children were mixed on what method of presentation children younger than themselves would need, and the difference did not rise to the level of significance. This indicates that the pre-service teachers held stronger beliefs that developmental processes underlie the ability to process complex science diagrams.

One interesting area where pre-service teachers and middle school students disagreed was on how middle school students would learn best from multiple visual representations. While the pre-educators felt students would need serial presentation, $78.4 \%$ of students preferred simultaneous presentation and the ability to compare and contrast across related representations shown together. This mismatch between the beliefs that pre-service teachers held about student learning, and the students' own metacognitive beliefs may signal misunderstandings about learner capabilities. Pre-service teachers appear to take a cautious view of the limits of the EFs of students as they grapple with complex diagrams, while students may overestimate their abilities to make meaningful connections between related science representations.

A second study was designed to examine how different presentation styles affected student learning. The results of that study are summarized in the next section.

\section{STUDY 2: EXPERIMENT VARYING PRESENTATION OF VISUAL REPRESENTATIONS IN A MIDDLE SCHOOL LESSON ON MITOSIS AND MEIOSIS}

While the survey results of Study 1 suggest that both adults and children have deeply held beliefs about the ways students
TABLE 2. Participating school demographics

\begin{tabular}{lcc}
\hline Demographic & School A & School B \\
\hline Total student body & 771 & 555 \\
$\quad$ Student Participants in Study & 224 & 161 \\
Classes participating & 8 & 5 \\
African American & $13(2 \%)$ & $20(5 \%)$ \\
Asian & $399(52 \%)$ & $176(32 \%)$ \\
Hispanic/Latino & $53(7 \%)$ & $81(15 \%)$ \\
White & $238(31 \%)$ & $227(41 \%)$ \\
English language learners & $113(15 \%)$ & $98(18 \%)$ \\
Economically disadvantaged & $90(12 \%)$ & $123(22 \%)$ \\
Students with disabilities & $45(6 \%)$ & $67(12 \%)$ \\
\hline
\end{tabular}

learn from conceptually connected visual representations, the literature is not clear on how these beliefs align with actual learning outcomes. The second study provides data on student learning from two representations aligned in different ways. This study compared not only serial versus simple simultaneous diagram presentation, but also added two simultaneous presentation conditions suggested by cognitive scientists interested in EFs: simultaneous presentation with support for noticing and simultaneous presentation with structure mapping support.

\section{Methods}

Participants. The students in Study 1 also participated in Study 2 and were recruited through three seventh grade science teachers at two suburban schools. Both schools were from the same upper-middle-class district. Two of the teachers were from school A, and across their eight classes, they taught 224 of the study participants. The teacher at school B had five classes and a total of 161 study participants. Due to course work planning constraints of the teachers, researchers had only 1 day to collect data. Though no individual demographic data were collected, the students in the study group were described by participating teachers as representative of the school population, as summarized in Table 2.

The day before the study, students in all classes were given a letter to take home that described the study. The letter informed parents/guardians that students were not required to participate, and that no student-identifiable data would be collected. On the day of data collection, students were read a description of the study and indicated assent through the raising of hands. Students were informed that they could remove themselves from the study at any time and receive the same instruction through text-based instruction provided by their teacher. One student opted out of the study and was not included in further analyses.

This study was completed under the IRB approval of the University of California, Irvine, HS no. 2012-9111.

\section{Materials and Procedure}

Instructional Lesson. A computer-based instructional module was designed using the Web-based Inquiry Science Environment. The students first responded to a survey (described in Study 1) that asked them how they thought they would learn best from related diagrams. This was followed by a lesson that introduced the related concepts of cell replication and 
reproduction through mitosis and meiosis. The module forced students to complete learning tasks on each screen before moving forward. After advancing the module, they were not able to move backward. This ensured that students completed all steps of instruction in order.

Regardless of the method of presenting diagrams, the text of the lesson itself remained constant and was based on the printed life sciences textbook used by seventh-grade classrooms throughout the school district (Padilla, 2007). Five screens were included in mitosis instruction, one each for interphase, metaphase, anaphase/telophase, and cytokinesis. This aligned with the textbook presentation of the same material. Each screen included a diagram alongside the text. A sample of the instructional diagram for mitosis is provided in Figure 2.

After completing the mitosis instruction, students were given a constructed-response item that asked them to recall information from the lesson. This page did not include any diagrams, only a box that simply asked, "How would you describe the process of mitosis to a friend? Describe as many steps as you can." At the completion of this screen, students saw a graphic that praised them for their hard work.

The second segment of instruction introduced the concept of meiosis through a series of seven different screens: Introduction; Interphase; Prophase I; Metaphase I; Anaphase I and Telophase I; Cytokinesis I; and Meiosis II. These segments were designed to closely align with the mitosis screens in the module. The instructional text was adapted from the ninth-grade science textbook from the same publisher as the seventh-grade textbook (Miller and Levine, 2011). Some of the text was simplified to eliminate vocabulary to which the students had not yet been exposed and to match the instruction in the mitosis portion of the module. The meiosis diagram that appeared in the instructional module is shown in Figure 3.

Once students completed the meiosis instructional module, they again received a recall item on a screen containing only text. Similar to the prior recall item, students were asked how they would describe meiosis to a friend, describing as many steps as they could. Upon submission, students were provided a screen praising their hard work and their completion of this section.

Design. The experiment sought to test whether students' beliefs about learning from multiple representations were aligned with their patterns of learning from multiple representations. Specifically, the learning context was knowledge gain from conceptually related science diagrams rather than different diagrams of the same concept. The experimental manipulations therefore involved providing diagrams organized in four different ways within the lesson: 1) serial presentation of separate mitosis and meiosis diagrams; 2) simultaneous presentation of the diagrams side by side; 3) simultaneously presented diagrams that signaled the learner to key similarities and differences; and 4) simultaneously presented diagrams with support for structure mapping. Computer-generated random assignment to experimental condition was achieved within each classroom using dummy codes for each student, such that the researchers did not know which student was assigned which code or experimental condition. The classrooms were all existing, mixed-ability classes. Random assignment at the student level allowed us to minimize any effects of

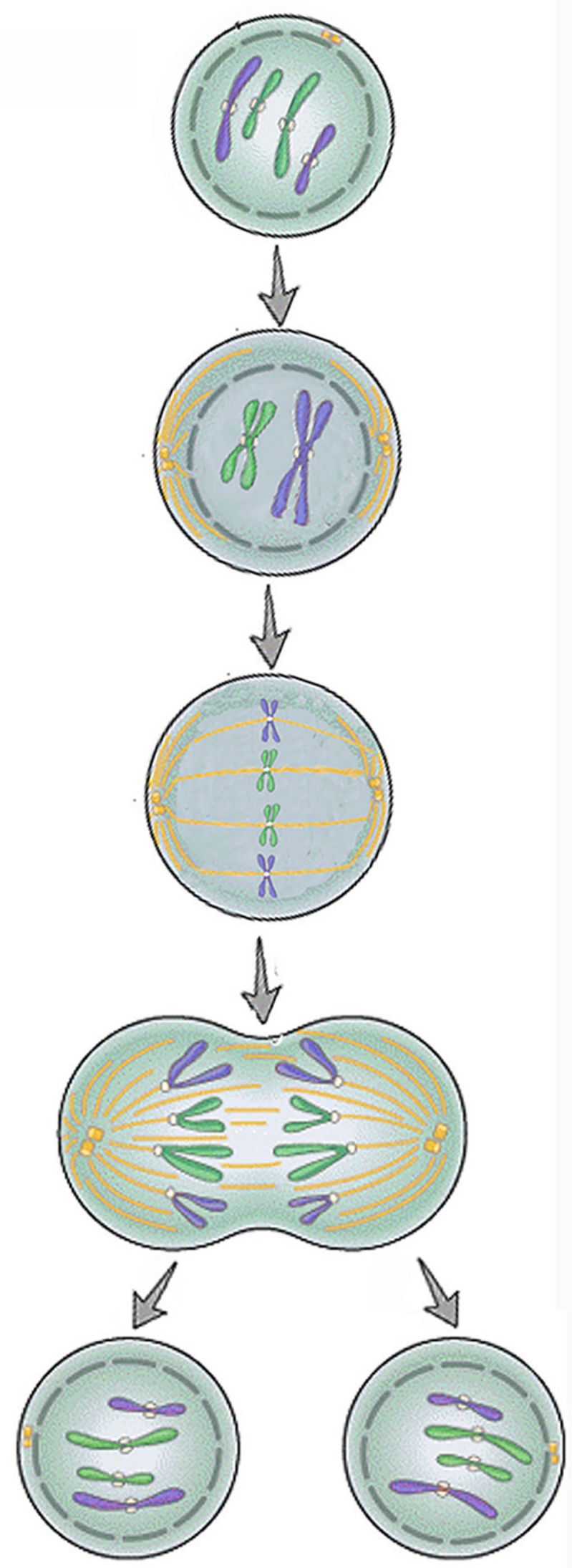

FIGURE 2. Mitosis diagram from instructional model. 


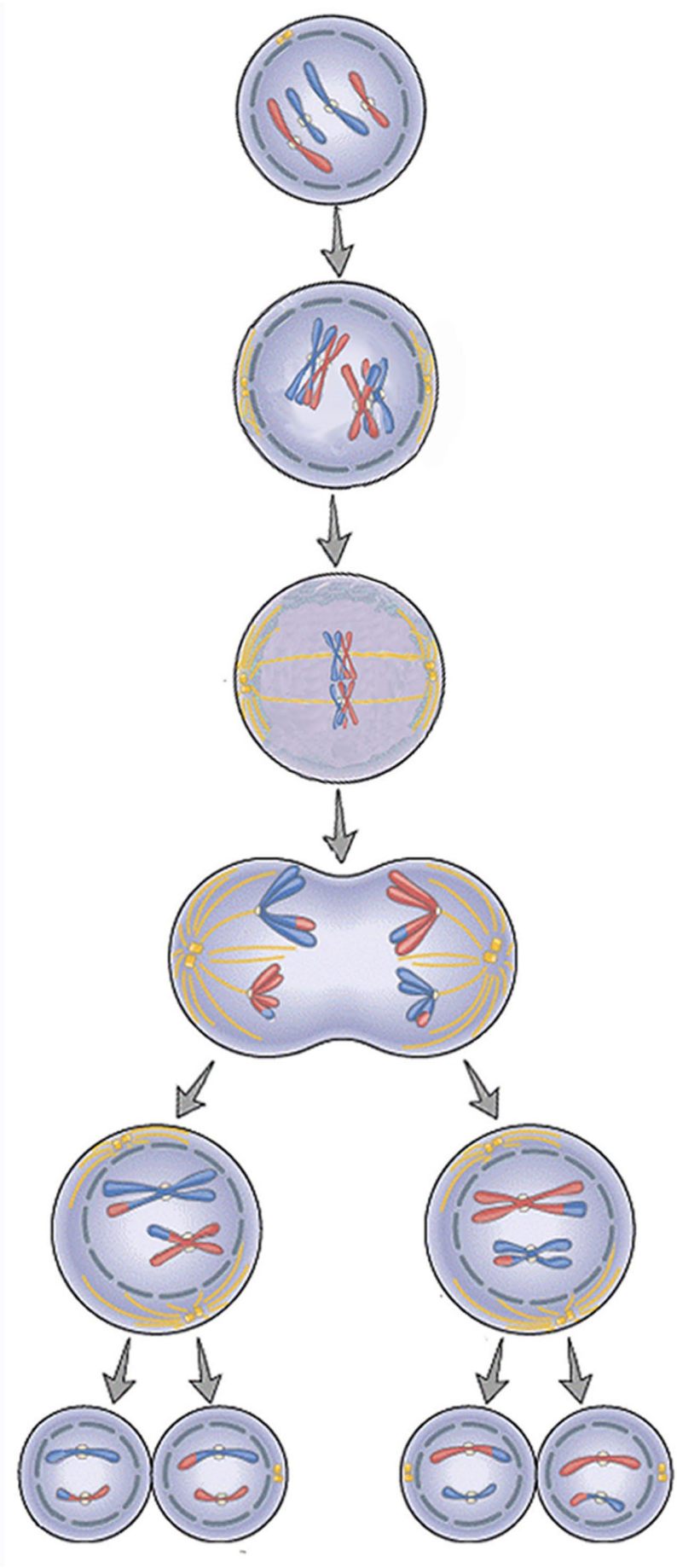

FIGURE 3. Meiosis diagram from instructional model.

classroom teacher or classroom-level characteristics and maximize ecological validity, as the instruction took place in a whole-classroom setting with peers and everyday social context. Written materials are provided in the Supplemental Material.

Serial Presentation. In the serial presentation condition, a mitosis diagram (see example in Figure 2) was provided to learners during all instruction related to learning about mitosis. A dia- gram of meiosis (see example in Figure 3) was provided to learners during all instruction related to meiosis. Diagrams were never shown on screen at the same time. No additional supports were provided. Serial presentation was included in all classrooms studied $(n=128)$.

Simultaneous Presentation. In the simultaneous presentation condition, a combined diagram showing mitosis and meiosis side-by-side (Figures 2 and 3 with initial cells aligned side-byside) was shown during all instruction. Therefore, when students were reading text about mitosis, they could also see the diagram for meiosis, and vice versa. There were no additional supports for noticing or interpreting the diagrams. Simultaneous diagrams were presented in all classrooms studied $(n=124)$.

Simultaneous with Signaling. In the simultaneous with signaling condition, students received the same combined diagram as in the simultaneous condition. The only difference was the addition of signaling prompts highlighted in red within the diagrams. These signals were designed to alert students to key features of the diagrams. For instance, when a diagram with instruction on cytokinesis was shown, red text asked, "Do the daughter cells look like the parent cells?" (see Supplemental Figure S1). This signaled learners to attend to an important phase in cell division that leads to miotic daughter cells that are identical to parents, while in meiotic cells, the daughter cells are each unique. Simultaneous diagrams with signaling were only offered at school A, with two teachers $(n=80)$.

Simultaneous with Structure Mapping Support. A fourth condition, simultaneous presentation with structure mapping support, draws on prior research that suggests that learners are better able to reason about representations with support (e.g., Gick and Holyoak, 1980, 1983) and better able to generalize their learning when actively participating in mapping the comparative relationships (Richland and McDonough, 2010). In this condition, learners received the screen that presented mitosis and meiosis simultaneously. However, before students left each instructional page, a mouse click would call up a question with a response box. For instance, the meiosis cytokinesis page read, "Take a close look at the picture, comparing the end of mitosis with the end of meiosis. In your own words, describe what is created by meiosis."

Active generation, or testing, is known to facilitate memory and retention (e.g., see Roediger and Karpicke, 2006), which suggests that by having students specifically generate alignments and comparisons, one can facilitate this learning. Similar to the signaling condition, students were alerted through highlighted text to key similarities or differences between the diagrams. But in addition to having their attention guided to the important element (signaling), students were asked to actively reason about what they were noticing, identifying the relationship between the diagrams themselves. Simultaneous diagrams with structure mapping support were only offered at school B $(n=53)$.

\section{Outcome Measures and Data Coding}

Outcome measures were derived from the free-response data written by students in response to prompts requesting students to describe mitosis and meiosis after instruction. This was 
designed to allow for a more nuanced understanding of the mental models of these systems that were developed by students, rather than simple accuracy rates in response to smaller, more explicit questions. Participant responses were downloaded directly from the teaching module into spreadsheet format for coding. Categorical codes were developed to quantify qualitative data coded by highly trained coders. At least two coders (including $\mathrm{JH}$ ) independently scored $20 \%$ of the data, yielding above $80 \%$ agreement (high to acceptable rates of agreement) using Cohen's kappa to control for chance reliability.

Descriptor codes for describing mitosis and meiosis were based on instructional text and iteratively refined through comparison to student responses at the development phase. Codes were derived from key principles within the biology of mitosis and meiosis, as well as characteristics of cognitive work that were predicted by the literature to indicate deep thinking, such as drawing connections and making inferences.

Mitosis. At the conclusion of mitosis instruction, all participants were asked to respond to the following prompt: "How would you describe mitosis to a friend? Fully describe as many steps as you can." Eight separate features when describing mitosis were identified. These features, along with their interrater reliability (kappa) score, are listed in Table 3.

Additional codes were added for "9: misconception" (e.g., "the male sperm and female egg meet"; $\mathrm{K}=0.95$ ), and "10: identification of surface features" ( $\mathrm{K}=0.81$ ) such as size (e.g., "The process keeps ... dividing into smaller parts"), color (e.g., "attached to a yellow string"), or nonspecific use of diagram labels (e.g., "It goes through interphase, prophase, metaphase, anaphase, telophase, and cytokinesis") or references stages (e.g., "I learned that mitosis is a process that has lots of steps to the cycle") as the whole response.

Meiosis. At the end of meiosis instruction, all participants were presented with a constructed-response item that asked: "How would you describe meiosis to a friend? Fully describe as many steps as you can." Student responses mentioned 10 different structural features of meiosis, shown in Table 4, along with their interrater reliability (kappa) statistic.

As with mitosis, coders scored when students mentioned surface features $(\mathrm{K}=1.0)$, like size or color, or simply listed names of phases rather than describing them. Some students mistakenly described the cell replication process of mitosis when responding to the "describe meiosis" prompt, and these responses were coded separately as well $(\mathrm{K}=0.90)$.

TABLE 3. Features of mitosis described and interrater reliability for each code

\begin{tabular}{lc}
\hline Feature described & Kappa \\
\hline Growth during interphase & 0.82 \\
Division & 0.84 \\
Creation of identical cells & 0.80 \\
Cell membrane & 0.81 \\
Chromosomes & 0.84 \\
Spindle fibers & 0.95 \\
Centrioles & 0.92 \\
Centromeres & 1.0 \\
\hline
\end{tabular}

TABLE 4. Features of meiosis described and interrater reliability for each code

\begin{tabular}{lc}
\hline Feature described & Kappa \\
\hline Growth during interphase & 0.82 \\
Division & 0.90 \\
Second division in meiosis & 1.0 \\
Cell membrane & 0.86 \\
Chromosomes & 0.84 \\
Spindle fibers & 0.84 \\
Centrioles & 0.82 \\
Centromeres & 0.85 \\
Crossing over & 0.93 \\
Non-identical cell creation & 0.83 \\
\hline
\end{tabular}

Principal Component Analyses. Principal component analysis for categorical data of the characteristics of mitosis and for meiosis was used to identify underlying patterns of responses. These analyses were completed using the Statistical Package for Social Sciences (SPSS) software. Principal component analysis was appropriate, as all data were categorical. Direct oblimin rotation was applied. An oblique rotation was preferred, as the individual components all refer to parts of the same process of cell division, and therefore correlation among variables was expected. Each component met the Kaiser criterion (Kaiser, 1960) for selection with an eigenvalue greater than 1.0. Component loadings greater than 0.40 were retained.

\section{Results}

Students' free responses to the "describe mitosis" and "describe meiosis" prompts provide data not only about student understanding of each process, but also on their inference errors across conditions.

Mitosis Free-Response Analyses. All cell features and cell processes noted by students were included in the principal component analysis. The descriptors clustered into three factors: rich description, which explained $29.05 \%$ of variance in the data; simple description, which explained $14.62 \%$ of the variance; and surface-level description, which explained $12.64 \%$ of total variance. Taken together, these factors explained $56.32 \%$ of variance in participant responses. The individual component loadings are described in Table 5.

Rich responses, factor 1 , meant that participants discussed several of the key features of mitosis and highlighted the role of spindle fibers and chromosomes. These can be contrasted with simple responses, factor 2 , which were responses that focused primarily on the cell growing and dividing, with little additional meaningful detail. Further, identification of identical cell creation as a feature of mitosis was negatively correlated within a simple response (see Table 5). Surface-level descriptions, factor 3, showed reliance on colors, shapes, sizes, or the use of labels without describing the process of replication or the creation of identical cells. These are important, because they reflect responses that are purely descriptive of the appearance of the diagrams and fail to engage in the abstract structure that is key to cell reproduction. These are responses that suggest the learner has not engaged in the higher-order, relational thinking that was intended in the instruction. Examples of each type of response are shown in Table 6. 
TABLE 5. Component loadings for "How would you describe mitosis to a friend?"a

\begin{tabular}{lccc}
\hline & $\begin{array}{c}\text { Rich } \\
\text { description }\end{array}$ & $\begin{array}{c}\text { Simple } \\
\text { description }\end{array}$ & $\begin{array}{c}\text { Surface-level } \\
\text { description }\end{array}$ \\
\hline Spindle fibers & $0.73^{\mathrm{b}}$ & & \\
DNA/chromosomes & 0.71 & & \\
Membrane & 0.64 & & \\
Centrioles & 0.54 & & 0.47 \\
Cell division & 0.40 & 0.62 & \\
Cell growth & & 0.63 & \\
Identical cell creation & & -0.50 & -0.51 \\
Surface features & & & 0.59 \\
\hline
\end{tabular}

${ }^{a}$ Component loadings $<0.40$ are suppressed.

bVariable principal normalization.

Regression scores for each component were obtained using SPSS and were then compared across conditions using a oneway between-subjects analysis of variance (ANOVA). There was no significant differences in the distributions of either rich description, $F(3,353)=0.34, p=0.80$, or simple description, $F(3,353)=1.33, p=0.27$, across condition, but there was an overall significant effect for surface-level description by condition, $F(3,353)=5.26, p=<0.01$.

To further understand group differences for relying on surface features in descriptions of mitosis, we conducted a series of $t$ tests for independent means of the regression scores. Participants in the signaling condition $(\mathrm{M}=0.24)$ were more likely to rely on surface features than those in the serial, $\mathrm{M}=-0.08, t(193)=$ $-2.86, p=<0.01$, or support for structure mapping, $M=0.00$, $t(119)=-0.24, p=<0.01$, conditions. Further, participants who received support for structure mapping outperformed those who saw the diagrams combined with no support, $\mathrm{M}=0.03, t(160)=$ $2.4, p=0.02$. These results are summarized in Table 7 .

Meiosis Free-Response Analyses. The same analysis was repeated for free-response data to a meiosis prompt. At the end of meiosis instruction, all participants were presented with a constructed-response item that asked: "How would you describe meiosis to a friend? Fully describe as many steps as you can." A total of 320 participants responded to this prompt, and this was coded as described in the study 2 Methods section.

Student responses were coded for mention of 10 different structural features of meiosis and for whether they made errors confusing meiosis for mitosis. These features are described more fully in Table 4.

These data were analyzed using principal component analysis to see whether and how each coded descriptor would contribute to overall patterns of responses. A total of three were identified: rich description, simplistic description, and confused with mitosis.

The three factors together explained $53.02 \%$ of variance in responses. Rich description explained 27.87\%; simplistic description explained 13.25\%; and confusing mitosis with meiosis explained $11.90 \%$ of variance in participant responses. The individual factor loadings are described in Table 8.

As with the "describe mitosis" prompt, we provide examples of the three patterns here. A rich description in answer to the "describe meiosis" prompt was associated with noting several different structural features of the replication process. A simplistic description might correctly identify meiosis as a process of cell division, but little else was fully described. While first and second cell division might be included, little more detail was described. The third type of

TABLE 6. Participant samples for "How would you describe mitosis to a friend?"

\section{Rich description}

I would describe mitosis to a friend as a process of making an identical cell. First, the cell makes a copy of the DNA. Two chromatin pair up, connected by a centromere, called chromosomes. The centrioles in the cell go to opposite ends, and create fibers, which are then stretched across the cell. The chromosomes line up along the center and the cell splits up, with the two daughter cells having the same amount of organelles and chromosome. A nuclear envelope forms around the cells.

\section{Simple description}

Mitosis is the process by which cells reproduce. This process involves three stages in which the cell enlarges, doubles in organelles, and essentially breaks apart forming several new cells.

\section{Surface-level description}

Example 1 (description relying on color): Inside the cell yellow things form. The yellow things push out and make two cells.

Example 2 (names phases without describing): That cells copy themselves to make new cells through interphase, prophase, metaphase, anaphase, telophase, and cytokinesis.

TABLE 7. Comparison of surface-level description of mitosis by condition: $t$ tests for independent means

\begin{tabular}{|c|c|c|c|c|c|c|}
\hline Condition & $n$ & Mean & SD & $t$ & $d f$ & $p$ \\
\hline Serial & 118 & -0.08 & 0.76 & 2.86 & 193 & $0.01 *$ \\
\hline Simultaneous with signaling & 77 & 0.24 & 0.74 & & & \\
\hline Simultaneous & 118 & 0.03 & 0.76 & 2.40 & 160 & $0.02 *$ \\
\hline Simultaneous with structure mapping & 44 & -0.29 & 0.72 & & & \\
\hline Simultaneous with signaling & 77 & 0.24 & 0.74 & 3.84 & 119 & $<0.01^{*}$ \\
\hline Simultaneous with structure mapping support & 44 & -0.29 & 0.72 & & & \\
\hline
\end{tabular}

*Denotes significant difference, $p<0.05$. 
TABLE 8. Component loadings for "How would you describe meiosis to a friend?"a

\begin{tabular}{lccc}
\hline & $\begin{array}{c}\text { Rich } \\
\text { description }\end{array}$ & $\begin{array}{c}\text { Simple } \\
\text { description }\end{array}$ & $\begin{array}{c}\text { Confused mitosis } \\
\text { with meiosis }\end{array}$ \\
\hline DNA/chromosomes & $0.68^{\mathrm{b}}$ & & \\
Spindle fibers & 0.67 & & \\
Centrioles & 0.62 & & -0.48 \\
Centromeres & 0.53 & & \\
Division: meiosis I & 0.53 & 0.58 & \\
Division: meiosis II & 0.52 & 0.53 & \\
Crossing over & 0.51 & & 0.49 \\
Membrane & 0.48 & & 0.66 \\
Cell growth & & & \\
Described mitosis & & & \\
\hline
\end{tabular}

${ }^{a}$ Component loadings $<0.40$ are suppressed.

bVariable principal normalization.

response identified by components analysis was one that confused mitosis with meiosis. Although the student may correctly identify cell growth, the defining factors of meiosis are not described. Examples of each type of response are shown in Table 9.

Regression scores for each of the three factors were calculated and compared across experimental condition using ANOVA. There were no significant differences in the distributions of either rich description, $F(3,316)=1.35, p=0.26$, or simplistic description, $F(3,316)=0.07, p=0.98$, responses across condition. There was, however, a significant condition effect for confusing meiosis with mitosis, $F(3,316)=3.43$, $p=$ 0.02 .

The error component of confusing mitosis with meiosis was isolated for further analysis across the data using $t$ tests for independent means. As shown in Figure 4, those in the simultaneous condition with structure mapping support $(\mathrm{M}=-0.32)$ were significantly less likely to be in the group that confused mitosis and meiosis than those in the serial, $(\mathrm{M}=0.00, t(133)$ $=2.5, p=0.01)$, simultaneous, $(\mathrm{M}=-0.01), t(140)=2.24, p=$ $0.03)$, or signaling, $(\mathrm{M}=0.15), t(107)=3.77, p<0.01)$, conditions.

\section{Discussion}

Like their adult non-educator counterparts, middle school children felt they would learn better from simultaneously presented visual representations of related science information. And, like the adults, they described the ability to compare and contrast across diagrams as desirable.

The experimental learning data provided insight into the validity of these beliefs and a more nuanced implication for instruction. While simultaneously presented representations did enhance student ability to make sense of science information concerning related concepts, they were only optimized when they included explicit supports for actively engaging learners in making the key connections across the representations. Simply having the two related diagrams presented together was not enough to engage and sustain the higher cognitive processes of the EF system, nor was directly drawing the learner's attention to key features of the diagrams. Middle school children did need support for the perceived benefit of comparison and contrast to be achieved

Though this experiment revealed that students in all conditions learned from instruction supported by visual representations, there were two important ways in which students in the supported structure mapping group outperformed the others. First, in describing mitosis, these students were far less likely to rely on surface features than those who received diagrams presented simultaneously either with no support or with only signaling support. They were not distracted by the number of steps, colors, shapes, or labels of the diagrams. Instead, active mapping of the correspondences appeared to draw attention to the processes rather than the drawings or the textual labels themselves.

Second, when presented with conceptually related science content, they were less likely to confuse the two processes, even though the diagrams were visible simultaneously during all instruction. This finding was robust, with the support for structure mapping group outperforming serial, simultaneous, and signaling conditions. It suggests that the students who received structure mapping support had a clearer picture of the key conceptual similarities and differences between the two cellular processes at the conclusion of instruction.

The greatest differences were seen when comparing the simultaneous presentation with signaling presentation and the structure mapping group. This is surprising, in that prior research has suggested that signaling can aid learners in identifying important aspects of complex diagrams. In the case of diagrams of related processes, however, adding only the written signals may have added too much to the overall cognitive load for learners whose EFs, without additional support, were not sufficient to handle both the simultaneous diagrams and the signals intended to direct their attention to key correspondences

TABLE 9. Participant samples for "How would you describe meiosis to a friend?"

Rich description

Meiosis is a process in which a parent cell makes four daughter cells that are all unique. In the first phase, chromatin are joined together at the centromere to make chromosomes. Next, the centrioles form spindle fibers that connect to the centromeres, pulling the chromosomes apart and leaving a little bit on each other. Then, they split into two daughter cells, each of which divide into two more daughter cells for a result of four unique daughter cells.

Simple description

The chromosomes intertwine and some DNA is swapped. The chromosomes are split and then they split again. Confused mitosis with meiosis

First, during meiosis, the cell makes copies of the DNA. Then, the chromosomes pair up and make duplicates. Later the chromosomes go to either end of the cell. Lastly, they will make exact copies of the cell. 


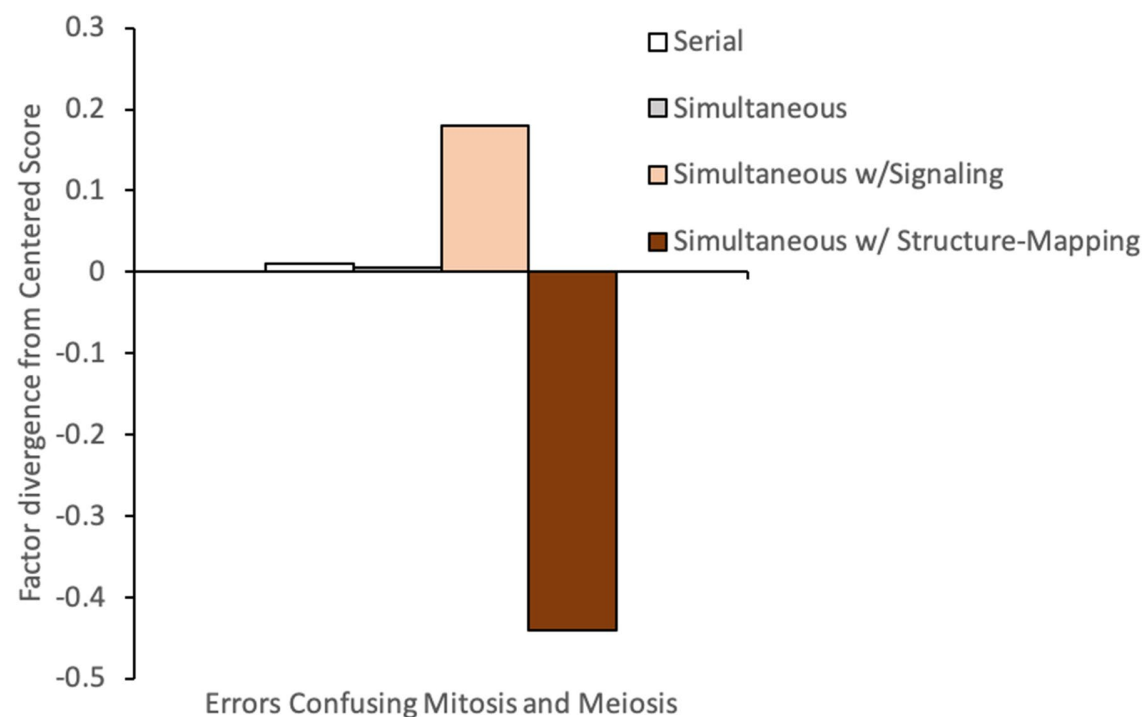

FIGURE 4. Mean component factor scores showing rates of errors confusing mitosis and meiosis across conditions.

(for a description of other research on outcomes related to signaling, see Mayer and Fiorella, 2019). This is contrasted with the support for structure mapping condition, which also included additional visual input, but actively engaged learners in describing what they saw instead of simply directing them to consider a specific aspect of the diagrams. Structure mapping prompts did appear to support learner ability to make sense of the same aspect of the diagram to which cueing only drew their attention. This is particularly notable, as the presence of both diagrams simultaneously did not overwhelm the learners in the supported condition, and it also appeared to enhance IC for information irrelevant to the task at hand.

\section{OVERALL DISCUSSION}

These studies elicited privately held beliefs from pre-service teachers, adult undergraduates, and middle school children about learning from visual representations of related science concepts and compared these beliefs to learning outcomes.

As predicted by prior research on EF development, and as shown in Figure 4, children did need support for mapping key elements across diagrams of the related science concepts of mitosis and meiosis in order to avoid surface-level understanding and errors confusing the represented ideas. But learners who received that support were able to develop more complex understandings of the key relationships between the two processes. This brings to light a possible misalignment between the beliefs of pre-service teachers, who as seen in Figure 1, endorsed serial presentation as easier for students to understand, and the metacognitive beliefs of children, who preferred the challenge of comparing and contrasting. While the pre-service teachers may be underestimating the EF of children's minds, children may overestimate what they can do without support.

Consistent with theory based in the relational reasoning literature (see Richland and McDonough, 2010; Begolli et al., 2018), this study found that children were able to process and create a deeper understanding of complex, related science topics when they had support for making connections across representations. This active involvement in making connections led to a lessened reliance on surface features of diagrams. More importantly, supporting students in structure mapping across related representations led to a deeper understanding of the key similarities and differences of the science concepts described and fewer misconceptions confusing mitosis with meiosis. The conditions that included active generation during learning may also have received a boost through the generation process supporting memory itself (e.g., see Roediger and Karpicke, 2006).

These studies show that focusing on addressing only the limitations of WM by limiting the presentation of simultaneous visual representations may lead to missed opportunities to help learners develop more complex mental models. Providing students with simultaneous representations of related science concepts can lead to learning that relies on structural correspondences rather than featural similarities and differences, but only if adequate support for EFs is provided. That these results held true in a real instructional setting with child learners is exciting, as they suggest that, when presented with support for mapping key relations, simultaneously presented visual representations of related science concepts can help students in science classrooms develop a greater understanding of complex interconnections in science.

\section{Limitations}

The main limitation of this study was the brevity of the overall delay to test. The constrained design allowed us high control in order to examine the effects of varying the instructional order and support for presenting materials. At the same time, it will be important to follow this work with an examination of how these effects persist over time.

Additionally, researchers were only able to test three conditions in each school. Though assignment was randomized across classrooms within those schools, comparison directly across schools was not possible for every condition. This may have underestimated school-level effects. Future studies would be further enhanced by the collection of student demographic and pretest and posttest data that could not be collected in the current study.

\section{Implications for Practice}

Together, these results provide new insights into how to optimize student learning from visual representations, and they also provide science educators with an important lens through which to consider their beliefs and practices of using visualizations. Integrating the theories of relational thinking and EFs helps to clarify why teachers must go beyond simply providing multiple visual models, diagrams, or other types of representations in sequence. We can infer that students may learn the details being shown in the representations when presented 
serially, and perhaps retention could even be facilitated in that way by reducing the amount of information to attend to, thereby reducing the overall EF load. But to promote broader understanding of how concepts fit together or to recognize commonalities and differences, this presentation style may not be optimal. Students may struggle to align and connect the ideas from two representations and ideas presented serially, which will limit the inferences they can make and may lead to misconceptions or misunderstandings. Thus, this report demonstrates the utility of supporting students in deepening their understanding of biology concepts by simultaneously showing two representations that are intended to be compared.

To best support teachers in incorporating this into their practice, we must also take note of the beliefs data we found. As shown in Figure 1, these data in particular suggest that teachers would benefit from being shown the distinctions between their beliefs about their own learning and their beliefs about teaching their students. We found that pre-service educators tended to believe that they learn differently than their students, which is an extremely important point for teacher education and science education researchers to consider. People's intuitions about their own learning did mirror the results we found in favor of better learning through supported simultaneous presentation. But pre-service teachers' intuition was to teach child learners through serial presentation of diagrams. We know that educators' beliefs are powerfully related to practice, which means that interventions and educational reforms that do not align with beliefs can be very difficult to change (see Munby, 1984; Wallace 2014).

Rather than aiming to convince teachers that their beliefs about teaching are incorrect, it likely will be more productive to highlight how their beliefs about their own learning are more in line with student learning in this case. That being said, their teaching beliefs seem to highlight that more support can be needed for younger learners to notice and draw connections across visual representations, which is also demonstrated in our data. So the overall implication is that learning can be optimized by presenting related visual representations simultaneously, but with additional support to help learners identify the relevant correspondences and differences without overloading their cognitive systems. Adding prompts for students to discuss and connect what they notice between these visual representations was a particularly powerful strategy. This has implications for both classroom teaching and visual texts, such as textbook design.

\section{ACKNOWLEDGMENTS}

This work was conducted with support from the National Science Foundation (grant no. 32027447) and the Institute of Education Sciences, through R305A190467 to the University of Chicago and the University of California, Irvine. The opinions expressed are those of the authors and do not necessarily represent views of the institute, the National Science Foundation, or the U.S. Department of Education.

\section{REFERENCES}

Ainsworth, S. (2014). The multiple representation principle in multimedia learning. In Mayer, R. E. (Ed.), The Cambridge handbook of multimedia learning, (pp. 464-486). Cambridge: Cambridge University Press. Doi: $10.1017 /$ cbo9781139547369.024

Ainsworth, S., \& Newton, L. (2014). Teaching and researching visual representations: Shared vision or divided worlds? In Eilam, B., \& Gilbert, J.
(Eds.), Science teachers' use of visual representations. (Models and modeling in science education, Vol. 8, pp 29-49). Cham, Switzerland: Springer International Publishing.

Baddeley, A. D., \& Hitch, G. J. (1974). Working memory. In Bower, G. A. (Ed.), The psychology of learning and motivation (pp. 47-89). New York: Academic Press

Begolli, K. N., \& Richland, L. E. (2016). Teaching mathematics by comparison: Analog visibility as a double-edged sword. Journal of Educational Psychology, 108(2), 194-213. doi: 10.1037/edu0000056

Begolli, K. N., Richland, L. E., Jaeggi, S. M., Lyons, E. M., Klostermann, E. C., \& Matlen, B. J. (2018). Executive function in learning mathematics by comparison: Incorporating everyday classrooms into the science of learning. Thinking and Reasoning, 24(2), 280-313. doi.org/10.1080/13546783 .2018 .1429306

Cho, S., Holyoak, K. J., \& Cannon, T. D. (2007). Analogical reasoning in working memory: Resources shared among relational integration, interference resolution, and maintenance. Memory \& Cognition, 25(6), 1445-1455.

Cook, M. P. (2006). Visual representations in science education: The influence of prior knowledge and cognitive load theory on instructional design principles. Science Education, 90, 1073-1091.

Deslauriers, L., McCarty, L. S., Miller, K., Callaghan, K., \& Kestin, G. (2019). Measuring actual learning versus feeling of learning in response to being actively engaged in the classroom. Proceedings of the National Academy of Sciences USA, 116(39), 19251-19257.

Diamond, A. (2002). Normal development of prefrontal cortex from birth to young adulthood: Cognitive functions, anatomy, and biochemistry. In Stuss, D. T., \& Knight, R. T. (Eds.), Principles of frontal lobe function (pp. 466-503). Oxford, England: Oxford University Press.

Diamond, A. (2013). Executive functions. Annual Review of Psychology, 64 $135-168$.

Eilam, B., \& Gilbert, J. K. (2014). The significance of visual representations in the teaching of science. In Eilam, B., \& Gilbert, J. (Eds.), Science teachers use of visual representations (Models and modeling in science education, Vol. 8, pp 3-28). Cham, Switzerland: Springer International Publishing.

Engle, R. W. (2002). Working memory capacity as executive attention. Current Directions in Psychological Science, 11(1), 19-23. Doi.org/ 10.1111/1467-8721.00160

Friedrichsen, P., Driel, J. H. V., \& Abell, S. K. (2011). Taking a closer look at science teaching orientations. Science Education, 95(2), 358-376.

Gick, M. L., \& Holyoak, K. J. (1980). Analogical problem solving. Cognitive Psychology, 12(3), 306-355.

Gick, M. L., \& Holyoak, K. J. (1983). Schema induction and analogical transfer. Cognitive Psychology, 15(1), 1-38.

Greeno, J. G., Collins, A. M., \& Resnick, L. (1996). Cognition and learning In Berliner, D., \& Calfee, R. (Eds.), Handbook of educational psychology. New York, NY: Simon \& Schuster Macmillan Publishing.

Holyoak, J. (2012). Analogy and Relational Reasoning. In Holyoak, K. J., \& Morrison, R. G. (Eds.), The handbook of thinking and reasoning (pp. 234259). Oxford, England: Oxford University Press.

Kaiser, H. F. (19600). The application of electronic computers to factor analysis. Educational and Psychological Measurement, 20, 141-151.

Kirschner, P. A. (2002). Cognitive load theory: Implications of cognitive load theory on the design of learning. Learning and Instruction, 12, 1-10.

Krawczyk, D. C., Morrison, R. G., Viskontas, I., Holyoak, K. J., Chow, T. W., Mendez, M. F., ... \& Knowlton, B. J. (2008). Distraction during relational reasoning: The role of prefrontal cortex in interference control. Neuropsychologia, 46(7), 2020-2032.

Luft, J. A., \& Roehrig, G. H. (2007). Capturing science teachers' epistemological beliefs: The development of the teacher beliefs interview. Electronic Journal for Research in Science \& Mathematics Education. 11(2), 38-63.

Matthewson, J. H. (1999). Visual-spatial thinking: An aspect of science overlooked by educators. Science Education, 83(1), 33-54.

Munby, H. (1984). A qualitative approach to the study of a teacher's beliefs. Journal of Research in Science Teaching, 21(1), 27-38.

Mayer, R. E. (2019). Cognitive theory of multimedia learning. In Mayer, R. E. (Ed.), The Cambridge handbook of multimedia learning (pp. 43-71). Cambridge: Cambridge University Press. 
Mayer, R. E., \& Fiorella, L. (2019). Principles for reducing extraneous processing in multimedia learning: Coherence, signaling, redundancy, spatial contiguity, and temporal contiguity principles. In Mayer, R. E. (Ed.), The Cambridge handbook of multimedia learning (pp. 279-315). Cambridge: Cambridge University Press.

Miller, K. R., \& Levine, J. S. (2011). Prentice Hall Biology: California. Upper Saddle River, NJ: Pearson Prentice Hall.

Miyake, A., \& Friedman, N. P. (2012). The nature and organization of individual differences in executive functions: Four general conclusions. Current Directions in Psychological Science, 21(1), 8-14. doi: 10.1177/: 22773897

Miyake, A., Friedman, N. P., Emerson, M. J., Witzki, A. H., Howerter, A., \& Wager, T. D. (2000). The unity and diversity of executive functions and their contributions to complex "frontal lobe" tasks: A latent variable analysis. Cognitive Psychology, 41(1), 49-100.

Muijs, D., \& Reynolds, D. (2015). Teachers' beliefs and behaviors: What really matters? Journal of Classroom Interaction, 50(1), 25-40

National Academy of Sciences. (2000) How people learn: Brain, mind, experience, and school. Washington DC.

National Research Council. (2012). A framework for $K-12$ science education practices, crosscutting concepts, and core ideas. Washington, DC: National Academies Press.

Next Generation Science Standards Lead States. (2013). Next Generation Science Standards: For states, by states. Washington, DC: National Academies Press.

Padilla, M. J. (2007). Focus on life science: California. Upper Saddle River, NJ: Pearson Prentice Hall.

Pamuk, S., Sungur, S., \& Oztekin, C. (2016). A multilevel analysis of students science achievements in relation to their self-regulation, epistemological beliefs, learning environment perceptions, and teachers' personal characteristics. International Journal of Science and Mathematics Education, 15(8), 1423-1440. doi: 10.1007/s10763-016-9761-7

Phillips, L. M., Norris, S. P., \& Macnab, J. S. (2010). Visualization in mathematics, reading and science education (Vol. 5). New York, NY: Springer Science $\&$ Business Media.
Richland, L. E., \& McDonough, I. (2010). Learning by analogy: Discriminating between potential analogies. Journal of Contemporary Educational Psychology, 35(1), 28-43.

Roediger, H.L., \& Karpicke, J. D. (2006). Test-enhanced learning taking memory tests improves long-term retention. Psychological Science, 17(3), 249-255.

Roth, K. J., Druker, S. L., Garnier, M. L., Lemmens, M., Chen, C., Kawanaka, T., ... \& Gonzales, P. (2006). Teaching science in five countries: Results from the TIMSS 1999 video study (NCES 2006-0110). Washington, DC: National Center for Education Statistics.

Schnotz, W. (2019). Integrated model of text and picture comprehension. In Mayer, R. E. (Ed.), The Cambridge handbook of multimedia learning (pp. 72-103). Cambridge: Cambridge University Press.

Schnotz, W., \& Bannert, M. (2003). Construction and interference in learning from multiple representation. Learning and Instruction, 13, 141-156.

Simms, N. K., Frausel, R. R., \& Richland, L. E. (2018). Working memory predicts children's analogical reasoning. Journal of Experimental Child Psychology, 166, 160-177.

Smith, M. S., Hughes, E. K., Engle, R. A., \& Stein, M. K. (2009). Orchestrating discussions. Mathematics Teaching in the Middle School, 14(9), 548-556.

Stein, M. K., Engle, R. A., Smith, M. S., \& Hughes, E. K. (2008). Orchestrating productive mathematical discussions: Five practices for helping teachers move beyond show and tell. Mathematical Thinking and Learning, 10(4), $313-340$.

Sweller, J., van Merrienboer, J. G., \& Paas, G. W. C. (1998). Cognitive architecture and instructional design. Educational Psychology Review, 10, 251-296.

Wallace, C. S. (2014). The role of science teachers' beliefs in international classrooms. In Evans, R., Luft, J., Czerniak, C., \& Pea, C. Overview of the Role of teacher Beliefs in Science Education (pp. 17-31). Rotterdam: Sense Publishers. https://doi.org/10.1007/978-94-6209-557-1

Waltz, J. A., Lau, A., Gewal, S. K., \& Holyoak, K. J. (2000). The role of working memory in analogical mapping. Memory \& Cognition, 28(7), 1205-1212.

Zook, K. B. (1991). Effects of analogical processes on learning and misrepresentation. Educational Psychology Review, 3(1), 41-72. 